\title{
Flicker electroretinograms of eyes with cataract recorded with RETeval system before and after mydriasis
}

This article was published in the following Dove Press journal:

Clinical Ophthalmology

\author{
Gen Miura \\ Takayuki Baba \\ Toshiyuki Oshitari \\ Shuichi Yamamoto \\ Department of Ophthalmology and \\ Visual Science, Chiba University \\ Graduate School of Medicine. \\ Chiba, Japan
}

Correspondence: Gen Miura Department of Ophthalmology and Visual Science, Chiba University Graduate School of Medicine, Inohana I-8-I, Chuo-ku, Chiba 260-8670, Japan

Tel +8 I 432262124

Fax +8I 432244162

Email gmiura2@chiba-u.jp
Purpose: The aim of this study is to determine the effect of pupil size of eyes with cataracts on the flicker electroretinograms (ERGs) elicited and recorded with the RETeval system.

Patients and methods: Forty-one eyes of 41 patients (mean age, $76.5 \pm 7.3$ years) that had grade 2 nuclear or cortical cataract without any other abnormalities were studied. Flicker ERGs were recorded before and after mydriatic drops instillation. The ERGs were elicited by the white light delivered at the frequency of $28.3 \mathrm{~Hz}$ and intensities of 2, 8, and $32 \mathrm{Td}$-s. The amplitudes and the implicit times of the flicker ERGs before and after mydriasis were compared.

Results: There were no significant differences between the amplitudes before and after mydriasis ( $P=0.35,2$ Td-s; $P=0.31,8$ Td-s; $P=0.50,32$ Td-s). There were also no significant differences between the implicit times before and after mydriasis ( $P=0.86,2 \mathrm{Td}-\mathrm{s} ; P=0.98,8$ Td-s; $P=0.95$, 32 Td-s). The mean amplitudes and implicit times of the nuclear and cortical cataracts groups before the mydriasis were also not significantly different from those after mydriasis for all stimulus intensities.

Conclusion: The lack of significant differences in the amplitudes and the implicit times of the flicker ERG of cataractous eyes before and after mydriasis indicated that the RETeval flicker ERGs in cataractous eyes is less affected by the pupil diameter. With our earlier study, it was assumed that the effect of cataracts on the RETeval flicker ERGs was due to the opacity of the crystalline lens, and the influence of the cataract would not be reduced or increased by mydriasis.

Keywords: electroretinogram, ERG, flicker ERG, cataract, RETeval

\section{Introduction}

The RETeval ${ }^{\text {TM }}$ system (LKC Technologies, Gaithersburg, MD, USA) is a noninvasive, mydriatic-free electroretinographic (ERG) system that can elicit and record not only flicker ERGs but also ERGs elicited by different stimulus intensities. ${ }^{1}$ Therefore, the RETeval can be used for objective assessments of retinal function and conventional fullfield ERGs. The RETeval system is designed to screen diabetic retinopathy because it can be tested without mydriasis, and flicker ERGs can be recorded in a short time..$^{2-4}$

Because the prevalence of cataract is relatively high, the presence of cataracts is a problem especially when screening a large number of individuals. Therefore, information of the effect of cataracts on the flicker ERGs recorded by the RETeval system will contribute to improving the accuracy of diagnosing ocular disorders.

We have reported that the amplitudes and implicit times of the flicker ERGs recorded with the RETeval system were affected by cataracts. ${ }^{5,6}$ In these studies, we found that the amplitudes and implicit times of the flicker ERGs recorded with RETeval 
system at $8 \mathrm{Td}$-s were affected by cataracts, and more specifically, the mean amplitude was significantly smaller, and the mean implicit time was significantly longer in eyes with cataracts. We also found that the effects of crystalline lens opacities on the implicit time decreased as strength of the light intensity increased. The effect was essentially eliminated when the stimulus intensity was 32 Td-s. However, the limitation of these studies was that all of the patients were examined under mydriatic-free conditions. Although RETeval system measures the pupil size and keeps the retinal illuminance constant during the examination, it is not entirely known how the lens opacities affect the flicker ERGs if the pupil diameter and the distribution of the light stimulus on the entire retina are different.

Thus, the purpose of this study is to determine the effect of pupil size of cataractous eyes on the flicker ERGs recorded with various stimulus intensities with the RETeval system. The amplitudes and implicit times recorded before the mydriasis were compared to that recorded after the mydriasis. In addition, we divided the eyes into those with cortical cataracts and those with nuclear cataracts and investigated the effects of mydriasis on the amplitudes and implicit times.

\section{Patients and methods}

Forty-one eyes of 41 patients that had cataracts were studied at the Chiba University Hospital between November 2016 and July 2017. All of the patients had flicker ERGs recorded with RETeval system before and after mydriasis. The procedures used in this study conformed to the tenets of the Declaration of Helsinki and were approved by the Institutional Review Board of Chiba University Hospital (number 2075). Patients were informed on the purpose of the examinations, including mydriasis and ERG recordings, and that information collected during the examination could be used for present and future research studies, and a written informed consent was obtained from all patients.

All patients had complete ophthalmic examinations including measurements of the best-corrected visual acuity (BCVA), intraocular pressure, slit-lamp examinations, and indirect ophthalmoscopy examinations. The BCVA was measured monocularly using a Japanese standard Landolt ring chart (System Charts SC-2000 Nidek Instruments, Gamagori, Japan) at a distance of $5 \mathrm{~m}$. The decimal visual acuities were converted to the logarithm of the minimum angle of resolution (logMAR) units for the statistical analyses. Only patients with grade 2 cataract based on the Emery-Little classification ${ }^{7}$ were studied.

None of the patients had any other ocular abnormalities in the anterior segment, media, and fundus except cataract.
Patients with diabetes mellitus and with refractive errors (spherical equivalents) greater than \pm 6 diopters were excluded.

Flicker ERGs were recorded with the RETeval system (LKC Technologies) from all patients before and after mydriasis. We used $0.5 \%$ tropicamide and $0.5 \%$ phenylephrine (Sandol P; Nitten Pharmaceutical Co. Ltd., Aichi, Japan) to dilate the pupils. All ERGs were recorded after 30 minutes after mydriasis. The pupil size of all patients ranged from 2 to $4 \mathrm{~mm}$ during the ERG recordings before mydriasis, and only cases with a pupil diameter $\geq 8 \mathrm{~mm}$ after the mydriasis were included in this study.

Skin electrodes were used to pick up the ERGs that were elicited by white light delivered at the frequency of $28.3 \mathrm{~Hz}$ and intensity of 2, 8, and 32 Td-s. No background light was used. The contralateral eye was patched during the recordings. The amplitudes and implicit times of the fundamental component were automatically extracted and displayed by the RETeval system using an embedded algorithm.

Wilcoxon signed-rank tests were used to determine the significance of the differences in the amplitudes and implicit times of the flicker ERGs. A $P$-value of $<0.05$ was considered as statistically significant.

\section{Results}

We studied 41 eyes of 41 patients ( 21 men and 20 women) whose mean age was $76.5 \pm 7.3$ years with a range of 62-88 years. The mean BCVA was $0.35 \pm 0.31 \log$ MAR units. The mean pupil size before and after mydriasis were 2.33 and $8.56 \mathrm{~mm}$, respectively. Seventeen eyes had nuclear cataracts and 24 eyes had cortical cataracts (Table 1). Representative flicker ERGs elicited by intensity before and after mydriasis are shown in Figure 1.

For all eyes, the mean amplitude and implicit times before mydriasis were $2.7 \pm 1.3 \mu \mathrm{V}$ and $39.6 \pm 2.3 \mathrm{~ms}$ with 2 Td-s, 9.2 $\pm 3.7 \mu \mathrm{V}$ and $35.9 \pm 1.7 \mathrm{~ms}$ with $8 \mathrm{Td}-\mathrm{s}$, and $15.3 \pm 4.6 \mu \mathrm{V}$ and $31.7 \pm 1.8 \mathrm{~ms}$ with $32 \mathrm{Td}$-s. The mean amplitude and implicit times after mydriasis were $2.8 \pm 1.6 \mu \mathrm{V}$ and $39.7 \pm 2.2 \mathrm{~ms}$ with $2 \mathrm{Td}-\mathrm{s}, 9.0 \pm 4.7 \mu \mathrm{V}$ and $35.8 \pm 1.8 \mathrm{~ms}$ with $8 \mathrm{Td}-\mathrm{s}$, and $15.5 \pm 5.5 \mu \mathrm{V}$ and $31.9 \pm 2.3 \mathrm{~ms}$ with $32 \mathrm{Td}-\mathrm{s}$ (Tables 2 and 3). The mean amplitudes before mydriasis

Table I Demographics of patients studied

\begin{tabular}{ll}
\hline & $\mathbf{n}$ \\
\hline Number of eyes & 41 \\
Age (years) & $76.5 \pm 7.3$ \\
Sex (male/female) & $21 / 20$ \\
LogMAR visual acuity & $0.35 \pm 0.31$ \\
Types of cataract (cortical/nuclear) & $24 / 17$ \\
\hline
\end{tabular}

Note: Data are expressed as mean \pm SD.

Abbreviation: LogMAR, logarithm of the minimum angle of resolution. 

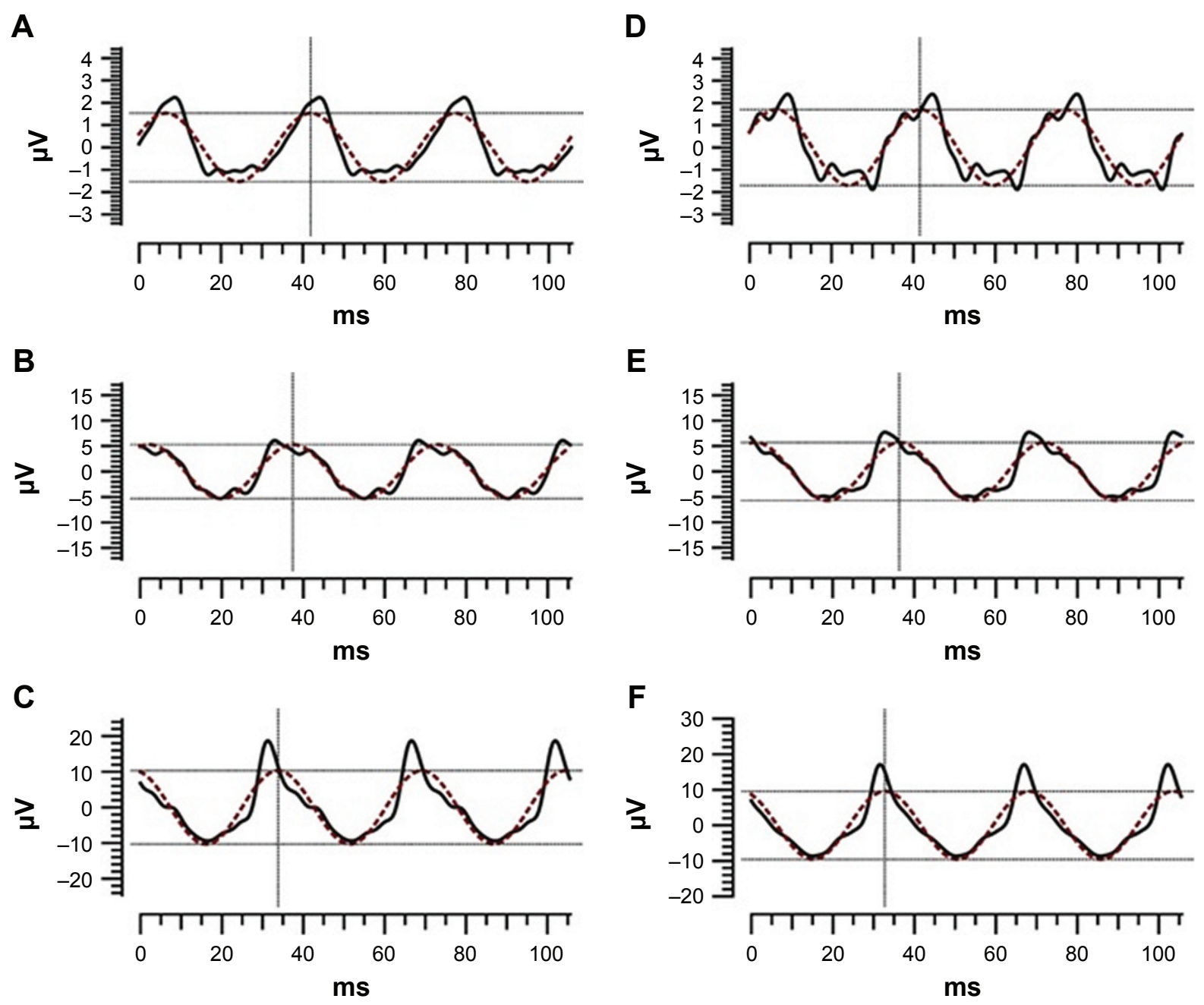

Figure I Representative flicker ERGs recorded with the RETeval system elicited by intensities of 2 (A, D), 8 (B, E), and 32 (C, F) Td-s before (A-C) and after (D-F) mydriasis.

Notes: Dotted lines are the superimposed fundamental components extracted by the RETeval system. Black lines are the actual waveforms. Abbreviation: ERG, electroretinogram.

were not significantly different from those after mydriasis for all stimulus intensities $(P=0.35,2$ Td-s; $P=0.31,8$ Td-s; $P=0.50,32 \mathrm{Td}-\mathrm{s})$. The mean implicit times before mydriasis were not significantly different from those after mydriasis for all stimulus intensities $(P=0.86,2 \mathrm{Td}-\mathrm{s} ; P=0.98,8 \mathrm{Td}-\mathrm{s}$; $P=0.95,32$ Td-s).

For eyes with cortical cataract, the mean amplitude and implicit times before mydriasis were $2.9 \pm 1.4 \mu \mathrm{V}$ and $39.2 \pm 2.1 \mathrm{~ms}$ with $2 \mathrm{Td}-\mathrm{s}, 10.0 \pm 4.2 \mu \mathrm{V}$ and $35.8 \pm 1.5 \mathrm{~ms}$

Table 2 Amplitudes of flicker ERGs recorded before and after mydriasis elicited by stimulus intensities of 2,8 , or $32 \mathrm{Td}-\mathrm{s}$

\begin{tabular}{llll}
\hline $\begin{array}{l}\text { Intensity } \\
(\text { Td-s) }\end{array}$ & $\begin{array}{l}\text { Before } \\
\text { mydriasis }(\mu \mathbf{V})\end{array}$ & $\begin{array}{l}\text { After } \\
\text { mydriasis }(\mu \mathbf{V})\end{array}$ & P-value \\
\hline 2 & $2.7 \pm 1.3$ & $2.8 \pm 1.6$ & 0.35 \\
8 & $9.2 \pm 3.7$ & $9.0 \pm 4.7$ & 0.31 \\
32 & $15.3 \pm 4.6$ & $15.5 \pm 5.5$ & 0.50 \\
\hline
\end{tabular}

Note: Data are expressed as mean \pm SD.

Abbreviation: ERG, electroretinogram. with $8 \mathrm{Td}$-s, and $15.7 \pm 5.1 \mu \mathrm{V}$ and $31.6 \pm 1.8 \mathrm{~ms}$ with $32 \mathrm{Td}$-s. The mean amplitude and implicit times after mydriasis were $2.8 \pm 1.8 \mu \mathrm{V}$ and $39.5 \pm 2.1 \mathrm{~ms}$ with $2 \mathrm{Td}-\mathrm{s}, 10.0 \pm 5.4 \mu \mathrm{V}$ and $35.7 \pm 1.7 \mathrm{~ms}$ with $8 \mathrm{Td}-\mathrm{s}$, and $16.2 \pm 6.1 \mu \mathrm{V}$ and $31.8 \pm 1.8 \mathrm{~ms}$ with 32 Td-s (Tables 4 and 5). The mean amplitudes before mydriasis were not significantly different from those after mydriasis for all stimulus intensities $(P=0.47,2$ Td-s; $P=0.67$, 8 Td-s; $P=0.35,32$ Td-s). The mean implicit times before mydriasis were not significantly different from those after

Table 3 Implicit times of flicker ERGs recorded before and after mydriasis elicited by intensities of 2, 8 , or $32 \mathrm{Td}$-s

\begin{tabular}{llll}
\hline $\begin{array}{l}\text { Intensity } \\
\text { (Td-s) }\end{array}$ & $\begin{array}{l}\text { Before } \\
\text { mydriasis (ms) }\end{array}$ & $\begin{array}{l}\text { After } \\
\text { mydriasis (ms) }\end{array}$ & P-value \\
\hline 2 & $39.6 \pm 2.3$ & $39.7 \pm 2.2$ & 0.86 \\
8 & $35.9 \pm 1.7$ & $35.8 \pm 1.8$ & 0.98 \\
32 & $31.7 \pm 1.8$ & $31.9 \pm 2.3$ & 0.95 \\
\hline
\end{tabular}

Note: Data are expressed as mean \pm SD.

Abbreviation: ERG, electroretinogram. 
Table 4 Amplitudes of flicker ERGs of patients with cortical cataract recorded before and after mydriasis elicited by stimulus intensities of 2,8 , or $32 \mathrm{Td}$-s

\begin{tabular}{llll}
\hline $\begin{array}{l}\text { Intensity } \\
(\text { Td-s) }\end{array}$ & $\begin{array}{l}\text { Before } \\
\text { mydriasis }(\mu \mathbf{V})\end{array}$ & $\begin{array}{l}\text { After } \\
\text { mydriasis }(\mu \mathbf{V})\end{array}$ & P-value \\
\hline 2 & $2.9 \pm 1.4$ & $2.8 \pm 1.8$ & 0.47 \\
8 & $10.0 \pm 4.2$ & $10.0 \pm 5.4$ & 0.67 \\
32 & $15.7 \pm 5.1$ & $16.2 \pm 6.1$ & 0.35 \\
\hline
\end{tabular}

Note: Data are expressed as mean \pm SD.

Abbreviation: ERG, electroretinogram.

mydriasis for all stimulus intensities ( $P=0.44,2$ Td-s; $P=0.90$, 8 Td-s; $P=0.98,32$ Td-s).

For eyes with nuclear cataracts, the mean amplitude and implicit times before mydriasis were $2.5 \pm 1.1 \mu \mathrm{V}$ and $40.0 \pm 2.6 \mathrm{~ms}$ with $2 \mathrm{Td}-\mathrm{s}, 8.1 \pm 2.6 \mu \mathrm{V}$ and $36.0 \pm 2.1 \mathrm{~ms}$ with 8 Td-s, and $14.7 \pm 3.9 \mu \mathrm{V}$ and $31.7 \pm 2.0 \mathrm{~ms}$ with $32 \mathrm{Td}-\mathrm{s}$. The mean amplitude and implicit times after mydriasis were $2.7 \pm 1.3 \mu \mathrm{V}$ and $40.0 \pm 2.2 \mathrm{~ms}$ with $2 \mathrm{Td}-\mathrm{s}, 7.6 \pm 3.0 \mu \mathrm{V}$ and $35.8 \pm 2.0 \mathrm{~ms}$ with $8 \mathrm{Td}-\mathrm{s}$, and $14.6 \pm 4.4 \mu \mathrm{V}$ and $32.2 \pm 2.9 \mathrm{~ms}$ with 32 Td-s (Tables 6 and 7). The mean amplitudes before mydriasis were not significantly different from those after mydriasis for all stimulus intensities $(P=0.49,2$ Td-s; $P=0.19$, 8 Td-s; $P=0.70,32$ Td-s). The mean implicit times before mydriasis were also not significantly different from those after mydriasis for all stimulus intensities $(P=0.48,2$ Td-s; $P=0.90,8$ Td-s; $P=0.99,32$ Td-s).

\section{Discussion}

Some earlier studies had shown that cataracts reduced the response density of multifocal ERGs (mfERGs) in the macular region but increased the peripheral mfERGs due to light scattering. ${ }^{8,9}$ Another study reported that different types of cataracts had different light scatter characteristics. ${ }^{10}$ Hence, we assumed that the effect of light scattering would be different if the pupil diameters were different in the ERGs recorded from cataractous eyes. These differences can then affect the ERG waveforms. However, our results showed that there were less significant differences between the amplitudes and implicit times before and after mydriasis in cases with grade 2 cataract. This indicates that even if the

Table 5 Implicit times of flicker ERGs of patients with cortical cataract recorded before and after mydriasis elicited by stimulus intensities of 2 , 8 , or $32 \mathrm{Td}$-s

\begin{tabular}{llll}
\hline $\begin{array}{l}\text { Intensity } \\
(\text { Td-s) }\end{array}$ & $\begin{array}{l}\text { Before } \\
\text { mydriasis }(\mu \mathbf{V})\end{array}$ & $\begin{array}{l}\text { After } \\
\text { mydriasis }(\mu \mathbf{V})\end{array}$ & $P$-value \\
\hline 2 & $39.2 \pm 2.1$ & $39.5 \pm 2.1$ & 0.44 \\
8 & $35.8 \pm 1.5$ & $35.7 \pm 1.7$ & 0.90 \\
32 & $31.6 \pm 1.8$ & $31.8 \pm 1.8$ & 0.98 \\
\hline
\end{tabular}

Note: Data are expressed as mean \pm SD.

Abbreviation: ERG, electroretinogram.
Table 6 Amplitudes of flicker ERGs of patients with nuclear cataract recorded before and after mydriasis elicited by stimulus intensities of 2,8 , or $32 \mathrm{Td}$-s

\begin{tabular}{llll}
\hline $\begin{array}{l}\text { Intensity } \\
(\text { Td-s })\end{array}$ & $\begin{array}{l}\text { Before } \\
\text { mydriasis }(\mu \mathbf{V})\end{array}$ & $\begin{array}{l}\text { After } \\
\text { mydriasis }(\mu \mathbf{V})\end{array}$ & $P$-value \\
\hline 2 & $2.5 \pm 1.1$ & $2.7 \pm 1.3$ & 0.49 \\
8 & $8.1 \pm 2.6$ & $7.6 \pm 3.0$ & 0.19 \\
32 & $14.7 \pm 3.9$ & $14.6 \pm 4.4$ & 0.70 \\
\hline
\end{tabular}

Note: Data are expressed as mean \pm SD.

Abbreviation: ERG, electroretinogram.

pupil diameter is changed, the effect of the cataract on the amplitudes and implicit times of the RETeval flicker ERGs is not changed.

Our previous study showed that the amplitudes and implicit times did not differ significantly between the two types of cataracts in the mydriasis-free recording conditions. In this study, we divided the eyes into those with cortical cataract and those with nuclear cataract and investigated the effects of mydriasis on the amplitudes and implicit times. The results showed that the mean amplitudes and implicit times of eyes with either nuclear or cortical cataract before mydriasis were not significantly different from those after mydriasis for all stimulus intensities. These results indicated that the differences in the type of cataracts and their effect on light scattering had no effect on the components of the flicker ERGs in the mydriasis conditions when examining the RETeval flicker ERGs.

The effect of pupil size on RETeval flicker waveform was reported in the previous study. ${ }^{11}$ The authors reported that the implicit times of the RETeval flicker ERGs in healthy subjects were significantly prolonged when the pupil was larger than $\sim 6.5 \mathrm{~mm}$. In our study, the mean implicit times before mydriasis were not significantly different from those after mydriasis, which is contrary to the other result. The exact reason for this discrepancy was not determined, but possible reasons might be differences in the age and the presence of the cataract because the earlier study examined healthy volunteers whose median age was 33 years. The authors presumed that the Troland values overestimate the effective retinal illumination in photopic ERG examination and that would be dimmer than the specified Troland value with dilated

Table 7 Implicit times of flicker ERGs of patients with nuclear cataract recorded before and after mydriasis elicited by intensities of 2,8 , or $32 \mathrm{Td}$-s

\begin{tabular}{llll}
\hline $\begin{array}{l}\text { Intensity } \\
\text { (Td-s) }\end{array}$ & $\begin{array}{l}\text { Before } \\
\text { mydriasis (ms) }\end{array}$ & $\begin{array}{l}\text { After } \\
\text { mydriasis (ms) }\end{array}$ & P-value \\
\hline 2 & $40.0 \pm 2.6$ & $40.0 \pm 2.2$ & 0.48 \\
8 & $36.0 \pm 2.1$ & $35.8 \pm 2.0$ & 0.90 \\
32 & $31.7 \pm 2.0$ & $32.2 \pm 2.9$ & 0.99 \\
\hline
\end{tabular}

Note: Data are expressed as mean \pm SD.

Abbreviation: ERG, electroretinogram. 
pupils because of the Stiles-Crawford effect. Based on their hypothesis and our results, light scattering due to cataract may offset the Stiles-Crawford effect in this study.

There are few limitations in this study. First, only flicker ERGs were investigated, and other types of ERGs, eg, fullfield, mfERGs, and focal ERGs were not studied. It should be remembered that the RETeval system can elicit and record ERGs with other parameters. The other types of ERGs can be elicited from the cone system, which are also affected by cataracts. Although the effect of cataract on the ERGs elicited from the rod system is not known, it is estimated that the influence of cataract on the waveform become larger when the light intensity is weak.

Second, the retinal illuminance of the stimulus was lower than the International Society for Clinical Electrophysiology of Vision (ISCEV) standard for flicker stimulation. ${ }^{12}$ The reason why we chose 2,8 , and $32 \mathrm{Td}$-s in this study was that 8 Td-s was the RETeval's default settings for flicker ERGs for nondilated eyes. The primary aim of this study was to investigate the impact of cataracts and pupil sizes at the flash illuminance near the default of $8 \mathrm{Td}$-s when examining the RETeval flicker ERGs. However, RETeval can record flicker ERGs conforming to the ISCEV standard using a stronger light intensity. Our previous study showed that as the stimulus intensity increases, the implicit times are less affected by a cataract. Thus, the influence on the waveform by cataracts is thought to be less, especially, for implicit time with stronger light intensity. If mydriasis is possible and it is not the purpose of detecting sensitive retinal ischemia, the examination with mydriasis and stronger flicker stimuli such as that of the ISCEV standard may reduce the influence of lens opacity on the ERGs. The effects of cataracts on the flicker ERGs elicited by stronger light intensities need to be studied.

Third, only subjects with grade 2 Emery and Little cataracts that are described as being "semisoft and yellowish white," which means that they were comparatively mild lens opacities, were studied. These grade 2 cataracts were studied because it was found that grade 2 cataracts had a significant effect on the amplitudes and implicit times of RETeval flicker ERGs. In our previous study, we also targeted subjects with grade 2 cataract. It is estimated that changes in the waveform will also increase if a more dense cataract is used; however, it is unknown how it differs from this result. Further investigations are needed.

\section{Conclusion}

The lack of significant differences in the amplitudes and the implicit times of the flicker ERG of cataractous eyes before and after mydriasis indicated that the RETeval flicker ERGs in cataractous eyes are less affected by the pupil diameter. It was assumed that the effects of cataracts on the RETeval flicker ERGs were due to the opacity of the crystalline lens, and the influence of the cataract would not be reduced or increased by mydriasis.

\section{Acknowledgment}

The authors thank Professor Emeritus Duco Hamasaki, Bascom Palmer Eye Institute, University of Miami, for his critical discussion and editing of the final manuscript.

\section{Author contributions}

GM contributed to study conception, study design, data acquisition, data analysis, statistical analysis, drafting, and revising. TB contributed to data analysis and statistical analysis. TO contributed to data analysis and statistical analysis. SY helped supervised physicians and contributed to data interpretation. All authors contributed toward data analysis, drafting and critically revising the paper and agree to be accountable for all aspects of the work.

\section{Disclosure}

The authors report no conflicts of interest in this work.

\section{References}

1. Asakawa K, Amino K, Iwase M, et al. New mydriasis-free electroretinogram recorded with skin electrodes in healthy subjects. Biomed Res Int. 2017;2017:8539747.

2. Maa AY, Feuer WJ, Davis CQ, et al. A novel device for accurate and efficient testing for vision-threatening diabetic retinopathy. J Diabetes Complications. 2016;30(3):524-532.

3. Fukuo M, Kondo M, Hirose A, et al. Screening for diabetic retinopathy using new mydriasis-free, full-field flicker ERG recording device. Sci Rep. 2016;8(6):36591.

4. Al-Otaibi H, Al-Otaibi MD, Khandekar R, et al. Validity, usefulness and cost of RETeval system for diabetic retinopathy screening. Transl Vis Sci Technol. 2017;6(3):3.

5. Miura G, Nakamura Y, Sato E, Yamamoto S. Effects of cataracts on flicker electroretinograms recorded with RETeval ${ }^{\mathrm{TM}}$ system: new mydriasis-free ERG device. BMC Ophthalmol. 2016;5(16):22.

6. Miura G, Sato E, Yamamoto S. Flicker electroretinograms recorded with mydriasis-free RETeval system before and after cataract surgery. Eye (Lond). 2017;31(11):1589-1593.

7. Emery JM, Little JH, editors. Surgical Techniques, Complications and Results. Phacoemulsification and Aspiration of Cataract. St Louis, USA: CV Mosby; 1979:45-48.

8. Chan HL, Siu AW, Yap MK, Brown B. The effect of light scattering on multifocal electroretinography. Ophthalmic Physiol Opt. 2002; 22:482-490.

9. Tam A, Chan H, Brown B, Yap M. The effects of forward light scattering on the multifocal electroretinogram. Curr Eye Res. 2004;28(1):63-72.

10. de Waard PW, IJSpeert JK, van den Berg TJ, de Jong PT. Intraocular light scattering in age-related cataracts. Invest Ophthalmol Vis Sci. 1992; 33(3):618-625.

11. Kato K, Kondo M, Sugimoto M, Ikesugi K, Matsubara H. Effect of pupil size on flicker ERGs recorded with RETeval system: new mydriasis-free fullField ERG system. Invest Ophthalmol Vis Sci. 2015;56(6):3684-3690.

12. McCulloch DL, Marmor MF, Brigell MG, et al. ISCEV standard for full-field clinical electroretinography (2015 update). Doc Ophthalmol. 2015;130(1):1-12. 


\section{Publish your work in this journal}

Clinical Ophthalmology is an international, peer-reviewed journal covering all subspecialties within ophthalmology. Key topics include: Optometry; Visual science; Pharmacology and drug therapy in eye diseases; Basic Sciences; Primary and Secondary eye care; Patient Safety and Quality of Care Improvements. This journal is indexed on

Submit your manuscript here: http://www.dovepress.com/clinical-ophthalmology-journal
PubMed Central and CAS, and is the official journal of The Society of Clinical Ophthalmology (SCO). The manuscript management system is completely online and includes a very quick and fair peer-review system, which is all easy to use. Visit http://www.dovepress.com/ testimonials.php to read real quotes from published authors. 